\title{
Mechanisms of refractive index modification during femtosecond laser writing of waveguides in alkaline lead-oxide silicate glass
}

\author{
F. Vega ${ }^{a)}$ and J. Armengol \\ Departament d'Òptica i Optometria, Universitat Politècnica de Catalunya, C/Violinista Vellsolà \\ 37 08222-Terrassa, Spain \\ V. Diez-Blanco, J. Siegel, and J. Solis \\ Laser Processing Group, Instituto de Óptica del Consejo Superior de Investigaciones Cientificas, \\ C/Serrano 121 28006-Madrid, Spain \\ B. Barcones and A. Pérez-Rodríguez \\ Departament d'Electrònica, Universitat de Barcelona, C/ Martí i Franquès 1 08028-Barcelona, Spain \\ P. Loza-Alvarez \\ Ultrafast Imaging Group, ICFO-Institut de Ciències Fotòniques, C/Jordi Girona 29, \\ 1-D 08034-Barcelona, Spain
}

(Received 31 January 2005; accepted 1 June 2005; published online 8 July 2005)

\begin{abstract}
We report on the mechanisms responsible for the formation of optical waveguides upon femtosecond laser irradiation of an alkaline lead-oxide silicate glass. MicroRaman spectroscopy and nonlinear fluorescence were employed to probe the local glass network structure and the formation of optically active defects respectively. At low laser pulse energies, the laser modified region is formed by a single light guiding region, whereas for pulses above $14 \mu \mathrm{J}$ the modified region is formed by a central dark zone, which does not guide light, accompanied by light guiding zones located in the surrounding of the dark one. This behavior is different from that observed in common silica glass systems but agrees with recent results obtained in phosphate and heavy metal oxide glasses. However, our results show that, unlike the latter glass, local densification of the glass occurs in the whole laser modified region, i.e., in the dark and the guiding zones. The suppression of light guiding in the dark region is explained by a high density of absorbing color centers. () 2005 American Institute of Physics. [DOI: 10.1063/1.1994927]
\end{abstract}

Femtosecond laser pulses have demonstrated their ability to induce permanent refractive index changes inside bulk glass at the focal point of the beam. This method relies on the use of nonlinear multiphoton absorption for coupling the laser energy into a transparent material and has allowed the photowriting of three-dimensional microstructures inside bulk glass. ${ }^{1,2}$ Results obtained in fused silica and several other silica based glass systems ${ }^{3}$ have shown that the laser irradiation induces a densification of the glass, and hence an increase in the refractive index within the focal volume, which allows one to guide light. However, recent results reported in phosphate glass, ${ }^{4}$ heavy metal oxide glass, ${ }^{5}$ crystalline $\alpha$-quartz, ${ }^{6}$ and poly(methylmethacrylate) Ref. 7 suggest that in these materials the main structural modification consists of an expansion of the focal volume accompanied by a lower local density and refractive index that does not support light guiding. However, it is worth noting that experimental evidence for the low density of the nonguiding region was only provided in Refs. 6 and 7. Regions of higher refractive index, where light guiding was shown to occur, are created in the surroundings as the result of the compression induced by the expansion of the central region.

Aside from structural changes, the induced refractive index modification may have positive contributions from laserinduced color centers and/or lattice defects. ${ }^{3,8}$ However, thermal annealing experiments carried out in fused silica and borosilicate glasses ${ }^{9}$ have shown that the thermal stability of

\footnotetext{
${ }^{a)}$ Electronic mail: fvega@oo.upc.es
}

the induced color centers is not consistent with that of the induced refractive index, thus indicating that the refractive index variation cannot be explained only in terms of the color center model.

In this letter, we show that fs irradiation of alkaline leadoxide silicate glasses leads to the densification of the irradiated zone, regardless of the pulse energy used. At low pulse energies, light guiding occurs through the central densified region of the waveguides. At high pulse energies, light guiding through the central densified region is suppressed because of the high density of absorbing defects that are formed there, whereas light guiding occurs in the surrounding region, also showing an increase in density. Local densification is detected by use of spatially resolved microRaman measurements while nonlinear fluorescence imaging is used to reveal the formation of laser-induced color center defects.

The glass used in this work is an alkaline silicate glass with a low concentration of lead oxide (K10, commercially available from Schott) (Ref. 10) which represents an intermediate choice between a pure silicate-based glass and a heavy metal oxide one. Its linear and nonlinear refractive indices at the $800 \mathrm{~nm}$ irradiation wavelength, as well as its energy gap are $n_{o}=1.5, n_{2}=3.1 \times 10^{-20} \mathrm{~W} / \mathrm{m}^{2}$, and $3.7 \mathrm{eV}$, respectively. ${ }^{11}$ Waveguides are written inside a glass block of $10 \times 10 \times 13 \mathrm{~mm}^{3}$, using a Ti:Sapphire fs oscillator/amplifier system which delivers $800 \mathrm{~nm}, 100$ fs laser pulses at $1 \mathrm{kHz}$ repetition rate. After attenuation, the irradiation beam is focused below the sample surface using a long working distance $(30.5 \mathrm{~mm})$ objective [10×, 0.26 numerical aperture 


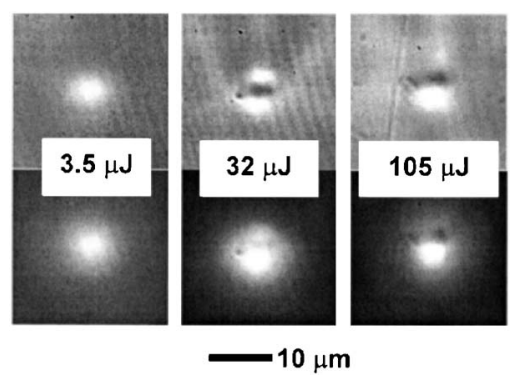

FIG. 1. Transillumination images of the exit face (upper row) and near-field images (lower row) obtained in three laser-written waveguides in K10 glass. The pulse energy used during the writing process is included in each image pair.

(NA)]. By means of a $X Y Z$ high precision translation stage, the sample is translated parallel to the laser beam at a constant speed of $100 \mu \mathrm{m} / \mathrm{s}$, thus writing longitudinal waveguides inside the glass block. In order to avoid surface damage during the writing process, the irradiation is initiated and stopped when the beam focus is $300 \mu \mathrm{m}$ underneath the entrance and exit planes of the glass block, respectively. Finally, the glass is cut and polished to bring the waveguide ends to the surface.

To study the optical performance of the waveguides, a $\mathrm{He}-\mathrm{Ne}$ laser beam is coupled into them by means of a microscope objective $(20 \times, 0.40 \mathrm{NA})$. Another objective ( 60 $\times, 0.85 \mathrm{NA}$ ) is used to image the near-field intensity distribution at the exit plane of the waveguide. By inserting a light diffuser in front of the coupling objective, the $\mathrm{He}-\mathrm{Ne}$ laser acts as an illumination source and transillumination images of the exit plane of the waveguides can be recorded, which allows one to determine the presence of laser-induced visible modifications. Figure 1 shows representative transillumination images and the corresponding near-field intensity distributions obtained from waveguides written at increasing laser pulse energies. The waveguide fabricated at the lowest pulse energy $(3.5 \mu \mathrm{J})$ exhibits a single bright region in the transillumination image. The near-field image shows that single mode light guiding occurs in the same region. This confirms that a refractive index increase has been induced throughout the glass block at the center of the laser irradiated region. A refractive index increase of $\approx 5 \times 10^{-4}$ is estimated from far field intensity distributions. A similar behavior is observed in all waveguides written up to pulse energies of $14 \mu \mathrm{J}$. Above this value, the transillumination images show a dark area in the central part of the laser modified region, which is surrounded by one or two bright areas. The deviation from an annular symmetry of the bright areas is most likely related to slight deviations from a circular symmetry of the writing laser beam profile. ${ }^{4,5}$

The microRaman measurements are carried out with a T64000 Jobin-Yvon spectrometer coupled to an Olympus metallographic microscope. The spectra are acquired in backscattering configuration using the $514 \mathrm{~nm}$ line of a continuous-wave argon-ion laser. The excitation beam is focused onto the glass surface by means of a microscope objective $(100 \times, 0.95 \mathrm{NA})$, which gives a submicrometric laser spot size at the sample surface. For the Raman measurements, excitation power at the sample site was $10 \mathrm{~mW}$. Figure 2(a) (curve labeled "bright") shows the spectrum recorded in the guiding region of a waveguide written at pulse energy below $14 \mu \mathrm{J}$. For the sake of comparison, a spectrum Downloaded 15 Feb 2010 to 161.111 .180 .191 . Redistribution subje
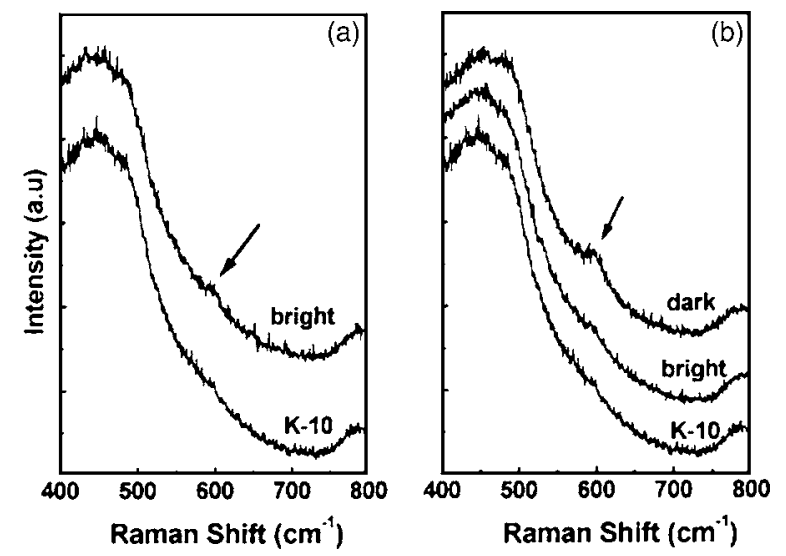

FIG. 2. MicroRaman spectrum obtained in the guiding region of a waveguide written at $3.5 \mu \mathrm{J}(\mathrm{a})$, and spectra obtained in the nonguiding and guiding regions of a waveguide written at $105 \mu \mathrm{J}(\mathrm{b})$. Spectra obtained in a nonirradiated region are included. All spectra are normalized to its maximum value and are vertically shifted for sake of clarity. The arrow indicates the $605 \mathrm{~cm}^{-1}$ peak.

obtained in nonirradiated glass is also included (curve labeled "K-10"). Both spectra show the broadbands corresponding to different $\mathrm{SiO}_{2}$ vibrational modes. In addition to these bands, the spectra also show a weak peak at about $605 \mathrm{~cm}^{-1}$, whose intensity is higher in the guiding region than in the nonirradiated one. This peak has been attributed in silica-based glasses to symmetric stretch breathing modes of the oxygen atom in a three-fold planar ring. ${ }^{12}$ An increase in peak intensity corresponds, therefore, to an increase in the relative number of these ring structures in the glass network. As a consequence, the overall bond angle distribution is reduced, thus leading to a densification of the glass. ${ }^{13}$ Since, according to the Lorenz-Lorentz relationship, densification implies a refractive index increase, we can conclude that the guiding regions correspond to laser-densified material.

Figure 2(b) shows the microRaman spectra obtained in a waveguide written with high pulse energy. The spectra are recorded in the central nonguiding region (curve labeled "dark"), the surrounding guiding one (curve labeled bright), and the unexposed material (curve labeled K-10). The comparison between these spectra shows that in the whole lasermodified region (i.e., central and surrounding regions) the intensity of the $605 \mathrm{~cm}^{-1}$ Raman peak is higher than in the nonirradiated area. Interestingly, the highest Raman peak intensity is achieved precisely in the central nonguiding region, which indicates that this region has the highest density.

To understand this, it is worth noting that active optical defects are often present when a laser-induced refractive index modification is induced. ${ }^{4,13-15}$ In order to detect the formation of color center defects, we have used an inverted Laser Scanning Microscope and a Ti:Sapphire laser that delivers pulses of $150 \mathrm{fs}, 800 \mathrm{~nm}$, at $76 \mathrm{MHz}$ repetition rate. The laser beam is sent to the glass surface through a microscope objective $(20 \times, \mathrm{NA}=0.45)$ and scanned by using two galvanometric mirrors. The nonlinear fluorescence emitted by the optically active defects at the glass surface is filtered through a broadband BG39 filter and detected by a charge coupled device camera. The nonlinear fluorescence is then recorded as a function of scanner position to form the image. Lastly, by recording the transillumination white light images of the scanned regions, it is possible to establish very accurately the position of the fluorescent defects with respect to to AlP license or copyright; see http://apl.aip.org/apl/copyright.jsp 


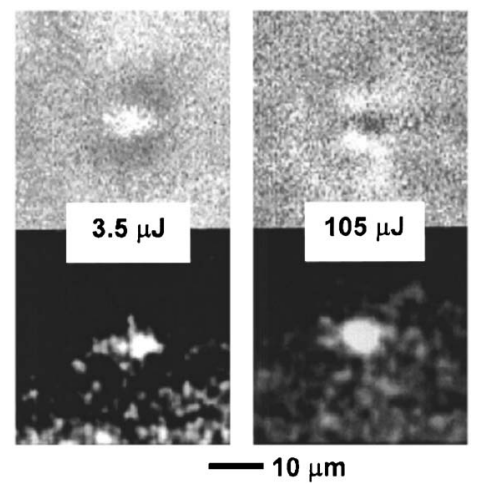

FIG. 3. Laser scanning transillumination images (upper row) and nonlinear fluorescence images (lower row) of the exit face obtained in two representative fs laser-written waveguides. The pulse energy used during the writing process is included in each image pair.

the region where the refractive index is modified. Figure 3 shows the transillumination and nonlinear fluorescence images obtained in two representative waveguides. In the case of waveguides written at pulse energies below $14 \mu \mathrm{J}$, the visible transformed region and the fluorescent one are similar in position, dimension, and shape, demonstrating that optically active defects are produced in the region of the glass that has experienced a laser-induced densification. According to the color center model, these defects might contribute to a refractive index increase in the visible and infrared through the Kramers-Kröning relationship. ${ }^{16}$

In the case of the waveguides that show a central nonguiding region, the fluorescence images reveal a high intensity signal from this region (Fig. 3), and thus the presence of a high density of color center defects, which might increase significantly the absorption. ${ }^{16}$ It can thus be concluded that the laser-densified dark areas are also highly absorbent ones which more likely impedes light guiding through this region of the waveguides.

In summary, fs laser writing of optical waveguides in alkaline lead-oxide silicate glass has been demonstrated. Waveguides written at pulse energies below $14 \mu \mathrm{J}$ show a single guiding region that is located at the center of the laser irradiated region. The mechanisms leading to the refractive index increase have been identified as structural densification accompanied by the formation of color center defects. For pulse energies above $14 \mu \mathrm{J}$, the waveguides present a central dark nonguiding region surrounded by guiding ones. We find that both, surrounding and central, regions show an increased density, as opposed to results reported for other systems, such as phosphate or heavy metal-oxide glasses. Our results provide evidence of a high density of absorbing defects which impedes light guiding through the dark region of the waveguides.

This work was partially supported by CICYT (Spain) under Project No. DPI2002-00151. One of the authors (V.D.B.) acknowledges the financial support of the CSIC and the European Social Fund through an I3P Ph.D. fellowship. Another author (P.L.-A.) acknowledges funding from the Spanish Government (the Ramón y Cajal program and Grant No. TIC2003-07485).

${ }^{1}$ K. Miura, J. Qiu, H. Inouye, T. Mitsuyu, and K. Hirao, Appl. Phys. Lett. 71, 3329 (1997).

${ }^{2}$ E. N. Glezer and E. Mazur, Appl. Phys. Lett. 71, 882 (1997).

${ }^{3}$ For an overview, see for instance, H. Ebendorff-Heidepriem, Opt. Mater. (Amsterdam, Neth.) 25, 109 (2004).

${ }^{4}$ J. W. Chan, T. R. Huser, S. H. Risbud, J. S. Hayden, and D. M. Krol, Appl. Phys. Lett. 82, 2371 (2003).

${ }^{5}$ J. Siegel, J. M. Fernández-Navarro, A. García-Navarro, V. Diez-Blanco, O. Sanz, J. Solis, F. Vega, and J. Armengol, Appl. Phys. Lett. 86, 121109 (2005).

${ }^{6}$ S. Nolte, M. Will, B. N. Chichkov, and A. Tünnermann, Proc. SPIE 4637, 188 (2002).

${ }^{7}$ A. Zoubir, C. Lopez, M. Richardson, and K. Richardson, Opt. Lett. 29, 1840 (2004).

${ }^{8}$ H. B. Sun, S. Juodkazis, M. Watanabe, S. Matsuo, H. Misawa, and J. Nishii, J. Phys. Chem. B 104, 3450 (2000).

${ }^{9}$ A. M. Streltsov and N. F. Borrelli, J. Opt. Soc. Am. B 19, 2496 (2002).

${ }^{10}$ The composition provided by Shott was (percentage by weight): Silica 70-80, lead oxide 1-10, sodium oxide 1-10, potassium oxide 1-10, and aluminum oxide 1-10.

${ }^{11}$ J. Solis, J. Siegel, J. M. Fernandez-Navarro, F. Vega, J. Armengol, V. Diez-Blanco, A. Garcia, and O. Sanz, Proc. SPIE 5662, 193 (2004).

${ }^{12}$ F. Galeener and A. E. Geissberge, Phys. Rev. B 27, 6199 (1983).

${ }^{13}$ J. W. Chang, T. Huser, S. Ribud, and D. M. Krol, Opt. Lett. 26, 1726 (2001).

${ }^{14}$ K. M. Davis, K. Miura, N. Sugimoto, and K. Hirao, Opt. Lett. 21, 1729 (1996).

${ }^{15}$ Y. Ikuta, K. Kajihara, M. Hirano, and H. Hosono, Appl. Opt. 43, 2332 (2004).

${ }^{16}$ C. Contardi, E. R. Taylor, and A. Fu, J. Non-Cryst. Solids 291, 113 (2001). 